\title{
Stability of Switched Linear Systems on Non-uniform Time Domains
}

\author{
John M. Davis \\ Dept. of Mathematics \\ Baylor University \\ Waco, TX 76798 \\ Email: John_M_Davis@baylor.edu
}

\author{
Ian A. Gravagne \\ Robert J. Marks \\ John E. Miller \\ Dept. of Elec. and Comp. Engineering \\ Baylor University \\ Waco, TX 76798 \\ Email: Ian_Gravagne@baylor.edu \\ Robert_Marks@baylor.edu \\ John_Miller1@baylor.edu
}

\author{
Alice A. Ramos \\ Dept. of Mathematics \\ Bethel College \\ Mishawaka, IN, 46545 \\ Email: alice.ramos@bethelcollege.edu
}

\begin{abstract}
A recent development in Lyapunov stability theory allows for analysis of switched linear systems evolving on nonuniform, discrete time domains. The analysis makes use of an emerging mathematical framework termed dynamic equations on time scales. We will present stability conditions for a general, arbitrarily switched system and then for system with a "constrained" switching signal. The results take the form of a compute-able inequality, which imposes conditions on the time domain itself.
\end{abstract}

\section{INTRODUCTION}

A number of methods have been developed to determine stability of linear systems [1], [2], [3], which can be applied to systems evolving on either continuous or discrete uniform time domains ( $\mathbb{R}$ or $\mathbb{Z}$, respectively). While these are valuable tools, there is an increasing interest in non-uniform time domainsdomains which contain non-uniformly spaced discrete points or a mixture of discrete and continuous parts [4]. Applications include adaptive control [5], real-time communications networks [6], [7], dynamic programming [8], switched systems [9], control theory [10], [11], [12], [13], [14], [15], signal analysis [16], [17], stochastic models [18], population models [19], and economics [20], [21].

Our interest is a specific class of linear systems called switched systems, where the overall dynamics are described using a number of sub-systems (modeled by continuousor discrete-time equations) coupled with discrete switching events. Because of this coupling, these (and other hybrid systems) are ideal candidates for time scales analysis.

One of the primary methods for determining stability of linear systems is the Second (Direct) Method of Lyapunov. His method can also be applied to switched systems using a common Lyapunov function [22]. The results presented in this paper extend the well-known results to nonuniform discrete time domains.

The rest of this paper will be structured as follows. In the next section, we give a short introduction to time scales calculus. We then present results for a general, arbitrary switching case. These results are then applied to a constrained switching case and some examples are presented. It is not possible to include all of the details for the results presented here, but the details and proofs are given elsewhere [23], [24], [25].

\section{TIME SCALES}

This section gives a brief outline of portions of the time scales theory which are particularly relevant to the following discussion. Readers are referred to several other sources to find a quick tutorial [26] or a more comprehensive introduction [27], [28], [29].

Time scales are generalized time domains and are denoted, in general, by the symbol $\mathbb{T}$. As mentioned above, the bulk of engineering systems theory to date rests on two time scales, $\mathbb{R}$ and $\mathbb{Z}$ (or more generally $h \mathbb{Z}$, meaning discrete points separated by distance $h$ ). These time domains give rise to the standard differential and difference equations, while the mathematical representation of a system evolving on a general time scale $\mathbb{T}$ is termed a dynamic equation.

The forward jump operator is given by $\sigma(t):=\inf _{s \in \mathbb{T}}\{s>$ $t\}$, while the backward jump operator is $\rho(t):=\sup _{s \in \mathbb{T}}\{s<$ $t\}$. The graininess function $\mu(t)$ is given by $\mu(t):=\sigma(t)-t$. Note that the graininess is analogous to the step size for a discrete time scale and on $h \mathbb{Z}, \mu(t)=h \forall t$. On $\mathbb{R}, \mu=0 \forall t$.

Let $\mathbb{T}^{\kappa}:=\mathbb{T}-m$, where $m$ is the right-most point in the (finite) time scale $\mathbb{T}$. This is necessary to avoid $\sigma(t)$ (and consequently $\mu(t)$ ) being undefined at the end of the time scale.

The $\Delta$-differential operator is defined as

$$
x^{\Delta}(t):=\frac{x(\sigma(t))-x(t)}{\mu(t)},
$$

where $t \in \mathbb{T}^{\kappa}$ and the quotient is taken in the sense that $\mu(t) \rightarrow$ $0^{+}$when $\mu(t)=0$. This definition of the delta derivative (or Hilger derivative) is equivalent to the more formal definition, which can be found in [28].

We next define the Hilger complex numbers as

$$
\mathbb{C}_{\mu}:=\left\{z \in \mathbb{C}: z \neq-\frac{1}{\mu}\right\}
$$


for $\mu \in \mathbb{R}^{+}$(positive real numbers). Along with this, the Hilger circle is defined as

$$
\mathcal{H}_{\mu}:=\left\{z \in \mathbb{C}_{\mu}:\left|z+\frac{1}{\mu}\right|=\frac{1}{\mu}\right\}
$$

These are easiest to see graphically, as shown in Figure 1.

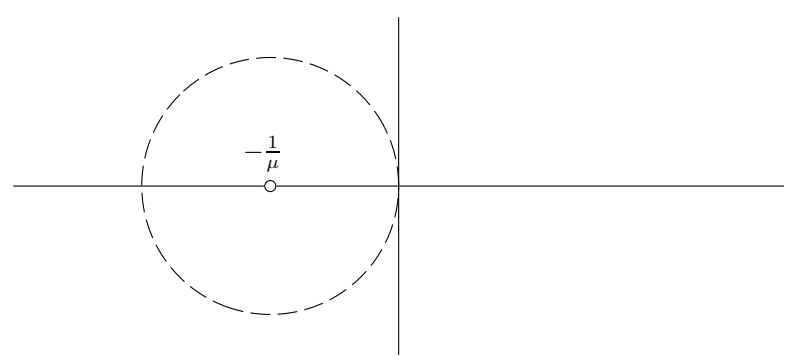

Fig. 1. The Hilger complex plane $\left(\mathbb{C}_{\mu}\right)$ and Hilger circle $\left(\mathcal{H}_{\mu}\right)$.

The function $p: \mathbb{T} \rightarrow \mathbb{R}$ is regressive if

$$
1+\mu(t) p(t) \neq 0, \quad \forall t \in \mathbb{T}^{\kappa}
$$

An $n \times n$ matrix-valued function $A(t)$ on a time scale $\mathbb{T}$ is regressive if

$$
I+\mu(t) A(t) \text { is invertible } \forall t \in \mathbb{T}^{\kappa} .
$$

Note that $A$ is regressive if and only if the eigenvalues, $\lambda_{i}(t)$, of $A$ are regressive for all $1 \leq i \leq n$.

\section{ARBITRARY SWITCHING}

Consider the time scales version of the typical switched LTI system composed of the set of subsystem matrices $A_{1}, A_{2}, \ldots, A_{m} \in \mathbb{R}^{n \times n}$ and a switching signal $s: \mathbb{T} \rightarrow$ $\{1,2, \ldots, m\}$, where

$$
x^{\Delta}(t)=A_{s(t)} x(t), \quad t \in \mathbb{T}, t \geq 0 \text { and } x(0)=x_{0} .
$$

We make the following assumptions about this system and the underlying time scale:

A1 The switching signal $s$ is arbitrary over $\mathbb{T}$.

A2 The eigenvalues of all of the $A_{i}$ are strictly within the Hilger circle for all $t \in \mathbb{T}$. (This means each $A_{i}$ is stable with respect to the time scale $\mathbb{T}$.)

A3 Each $A_{i}$ is regressive. (Meaning that $\left(I+\mu(t) A_{i}\right)^{-1}$ exists $\forall t \in \mathbb{T}$.)

A4 The family $\left\{A_{i}\right\}$ is pairwise commutative, i.e. $A_{i} A_{j}=$ $A_{j} A_{i} \forall i \neq j$.

A5 $\mathbb{T}$ has the following properties: (i) $0 \in \mathbb{T}$, (ii) $\mathbb{T}$ is unbounded above, (iii) $\mathbb{T}$ has graininess $0<\mu_{\min } \leq$ $\mu(t) \leq \mu_{\max }$ for all $t \in \mathbb{T}$.

We choose to investigate the stability of (1) by defining a Lyapunov candidate

$$
V=x^{T} P x
$$

where $P=P^{T}>0$. According to Lyapunov's Second Method, we need

$$
\begin{aligned}
& V^{\Delta}<0 \\
\Rightarrow & A_{i}^{T} P+P A_{i}+\mu A_{i}^{T} P A_{i}+\left(I+\mu A_{i}^{T}\right) P^{\Delta}\left(I+\mu A_{i}\right)<0
\end{aligned}
$$

to ensure stability. For reasons given in [23], we set

$$
A_{i}^{T} P+P A_{i}+\mu A_{i}^{T} P A_{i}=-M_{i},
$$

where $M_{i}=M_{i}^{T}>0$. Substituting (3) into (2) yields

$$
\left(I+\mu A_{i}\right)^{T} P^{\Delta}\left(I+\mu A_{i}\right)-M_{i}<0, \quad \text { for } t \in \mathbb{T}
$$

For simplicity, we define

$$
L_{i}(\mu):=2 \operatorname{Re} \Lambda_{i}+\mu \Lambda_{i}^{*} \Lambda_{i}
$$

where $\Lambda_{i}$ is a diagonal matrix of the eigenvalues of the $A_{i}$. Note that (4) depends indirectly on $t$ through $\mu$ and $\mu^{\sigma}$. This gives rise to a Temporal Region of Stability (TRoS) defined by Theorem 2.1 from [25], which is repeated here.

Theorem III.1 (General TRoS). Under assumptions A1-A5, given a set of matrices $A_{i}=S^{-1} J_{i} S$ for $1 \leq i \leq m$ where the $J_{i}$ are Jordan epsilon form matrices with $J_{i}=\Lambda_{i}+N$ and $S$ is a simultaneous similarity transform, there exists a region $\mathcal{R} \in \mathbb{R}^{2}$ consisting of pairs $\left(\mu, \mu^{\sigma}\right)$ such that

$$
\prod_{k=1}^{m} L_{k}^{-1}(\mu) L_{k}\left(\mu^{\sigma}\right)>\left(I+\mu \Lambda_{i}^{*}\right)\left(I+\mu \Lambda_{i}\right),
$$

for $i=1, \ldots, m$ and $0<\mu_{\min } \leq \mu(t) \leq \mu_{\max }$ for all $t \in \mathbb{T}$.

\section{CONSTRAined SWITCHING}

\section{A. Motivation}

While the previous section focused on the general problem of system stability, this section will focus on a specific application of those results. We begin by noting that many industrial and automotive systems use discrete controllers, which communicate with various sensors and actuators via a single real time network. One common protocol is the Controller Area Network (CAN). We will use this as our motivation; although, the ideas presented here are not limited to CAN systems or real-time networks.

A real-time network is typically comprised of three types of nodes connected by a single bus: controllers, sensors, and actuators. A control loop can be implemented on the network, which at its most basic, involves a control node polling one or more sensors, the sensors responding, and the controller calculating new actuator values and transmitting these to one or more actuators. While this is a typical closed-loop feedback system with a specified sampling period, there are also sporadic high-speed, high-priority messages that need to be handled. These come from (often random) events which trigger a sensor, such as depressing the brake pedal in a car or a robot hand making contact with an object. They are termed high-speed because they have (possibly very tight) deadlines and must be processed within those deadlines. 
One method of handling these sporadic messages is to allow more time (i.e. increase the sampling period) between the periodic messages. This deceases the robustness of the control-loop, however. An alternative tactic is to use adaptive sampling, meaning we can adjust the sampling period "onthe-fly." This allows for short periods of high-speed sporadic traffic but maintains system integrity. This type of adaptive control is the focus of [6].

We begin by describing (mathematically) the system using the same discretization method as [6]. We then present the main theorem, which is very similar (as should be expected) to Theorem III.1. We conclude with some examples of systems and their corresponding TRoS's. While the results presented give sufficient conditions on the sampling periods in order to maintain system stabiltiy, we do not present a particular adaptive sampling techinique/scheme/law here.

\section{B. Problem Setup}

Consider the linear system of the form

$$
\begin{gathered}
\dot{x}=A x+B u, \quad A \in \mathbb{R}^{n \times n}, B \in \mathbb{R}^{n \times p} \\
u=K x, \quad K \in \mathbb{R}^{p \times n} .
\end{gathered}
$$

We can discretize this system (as was done in [6]) by

$$
x^{\Delta}(t)=\mathcal{A}(\mu(t)) x(t)
$$

where

$$
\mathcal{A}(\mu(t)):=\operatorname{expc}(\mu(t) A)(A+B K) .
$$

Designating a finite number of choices of $\mu$ yields the set $\left\{\mu_{1}, \mu_{2}, \ldots, \mu_{m}\right\} \in \mathbb{R}^{+}$and a corresponding set of matrices $\left\{\mathcal{A}_{1}, \mathcal{A}_{2}, \ldots, \mathcal{A}_{m}\right\} \in \mathbb{R}^{n \times n}$ with switching signal $c:\left\{\mu_{i}\right\} \rightarrow$ $\{1,2, \ldots, m\}$. The switched system is then

$$
x^{\Delta}(t)=\mathcal{A}_{c(t)} x(t), \quad t \in \mathbb{T}, t \geq 0 \text { and } x(0)=x_{0} .
$$

We make the following modifications to the assumptions given in the arbitrary case:

- The switching signal in A1 now chooses from a finite set (of $\mu_{i}$ ), and, in that sense, is constrained.

- All references to $A_{i}$ now refer to $\mathcal{A}_{i}$.

\section{Stability Under Constrained Switching}

Before presenting Theorem IV.1, we note that each $\left(\mu_{i}, \mu_{j}\right)$ pair is associated with a specific $\left(\mathcal{A}_{i}, \mathcal{A}_{j}\right)$ pair. This follows from the problem setup (A1) and yields a system which switches coefficients depending on the current graininess. This means we can change the behavior of the system (and the region of stability) based on both our choice (or what is given to us) of $A, B$, and $K$ as well as our choice of the $\mu_{i}$ 's.

Theorem IV.1 (Constrained TRoS). Under assumptions A1$A 5$, given a set of matrices $\mathcal{A}_{i}=S^{-1} J_{i} S$ for $1 \leq i \leq m$ where the $J_{i}$ are Jordan-epsilon form matrices with $J_{i}=\Lambda_{i}+N$ and $S$ is a simultaneous similarity transform, there exists a region $\mathcal{R}_{c} \in \mathbb{R}^{2}$ consisting of pairs $\left(\mu_{i}, \mu_{j}\right)$ such that

$$
\prod_{k=1}^{m} L_{k}^{-1}\left(\mu_{i}\right) L_{k}\left(\mu_{j}\right)>\left(I+\mu_{i} \Lambda_{i}^{*}\right)\left(I+\mu_{i} \Lambda_{i}\right),
$$

for all $i, j=1, \ldots, m$ and $0<\mu_{\min } \leq \mu_{i} \leq \mu_{\max }$ for all $t \in \mathbb{T}$.

\section{EXAMPLES}

Whereas in Theorem III.1 we can calculate continuous TRoS's with upper bounds defined by the equality of (5), in this case, continuous regions do not have any meaning, only discrete points corresponding to values for $\mu_{i}$. The following figures have black (or dark) +'s where the inequality of (6) is satisfied (i.e. "valid" points), and red (or light) o's where it is not. Similar to the general case, the upper limits on the TRoS plots are equal to $\mu_{\max }$.

We use MATLAB to generate random, commuting, nondiagonal $A, B \in \mathbb{R}^{3 \times 3}$ pairs, and then calculate $K$ (also in $\mathbb{R}^{3 \times 3}$ ) according to the method given in [25] and a user specified (but arbitrarily chosen) set of desired eigenvalues of $A+B K$. Each example has three distinct eigenvalues. The eigenvalues of $A$ have a positive real part, while the desired (and actual) eigenvalues of $A+B K$ have a negative real part. Note that all of the following figures are most easily viewed in color.

\section{A. Example 1}

Figure 2 is a plot of the eigenvalues of $A$ and $A+B K$ and the Hilger circle corresponding to $\mu_{\max }$ for $A+B K$. The desired eigenvalues were $[-1.9,-1.2 \pm 0.3 i]$. Figures 4,5 , and 6 show that the region changes based on choices of $\mu_{i}$, both the number of choices and the values of those choices. For example, $(0.3,0.45)$ (approximately) is not a valid point in either of the first two plots, but is valid in the third. Figure 3 shows the eigenvalues of the $\mathcal{A}_{i}$ 's and corresponds to Figure 4. The smallest Hilger circle $\mathcal{H}_{\text {min }}$ (corresponding to $\mu_{\max }$ for the system) is also plotted in Figure 3 and encompasses all of the eigenvalues of the subsystems.

It is important to note that valid points above the $\mu_{i}=\mu_{j}$ line allow for more flexibility in the system. These points mean that the graininess can "upshift," or increase. With no valid points above the diagonal, the system is "trapped" and cannot increase the graininess. For example, if the system described in Figure 4 started with $\mu_{1} \approx 0.15$, it could only decrease the graininess (i.e. "downshift") or stay at approximately 0.15 . If it decreased, it could never return to 0.15 . The systems described by Figures 5 and 6, however, have complete freedom. Intermediate steps may be required, but they can upshift or downshift to reach every allowable graininess.

\section{B. Example 2}

Figures 7-11 are very similar to Example 1, but with a different $A, B$, and $K$. Note that the algorithm for calculating $K$ put the eigenvalues of $A+B K$ as close to the desired values $[-1.9,-1.2 \pm 0.3 i]$ as possible.

\section{CONCLUSION}

We have demonstrated a common Lyapunov function approach to stability of switched systems on a non-uniform, 


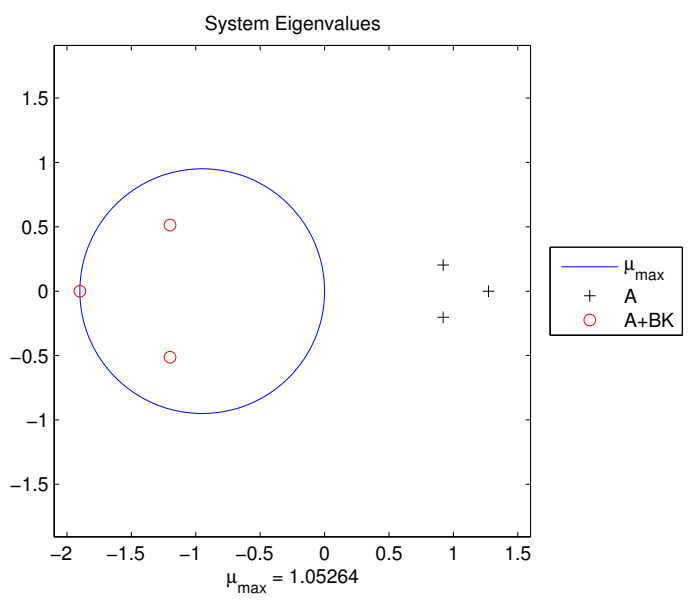

Fig. 2. Ex 1: Eigenvalues of $A$ and $A+B K$.

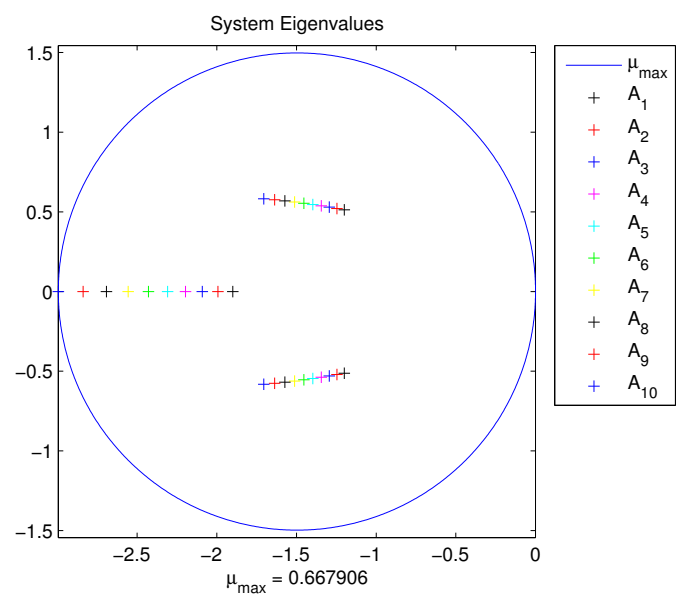

Fig. 3. Ex 1: Eigenvalues of $\mathcal{A}_{i}$ for $\mu_{i}<\mu_{\max }$ and $i=\{1, \ldots, 10\}$.

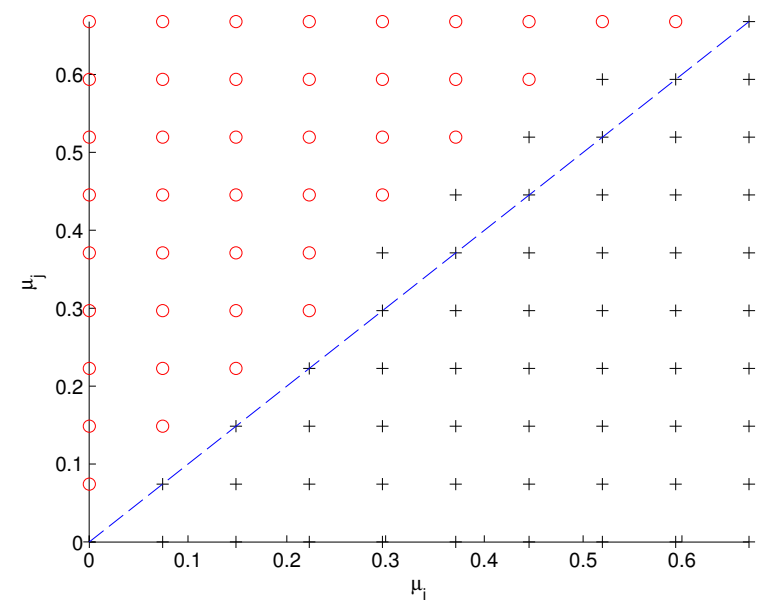

Fig. 4. Ex 1: $\mathcal{R}_{c}$ for $\mu_{i}<\mu_{\max }$ and $i=\{1, \ldots, 10\}$.

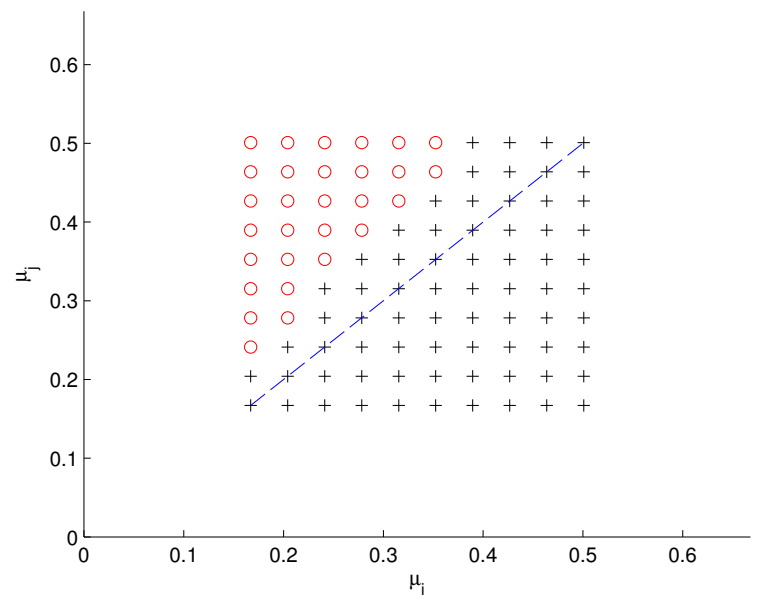

Fig. 5. Ex 1: $\mathcal{R}_{c}$ for $.25 \mu_{\max }<\mu_{i} \leq .75 \mu_{\max }$ and $i=\{1, \ldots, 10\}$.

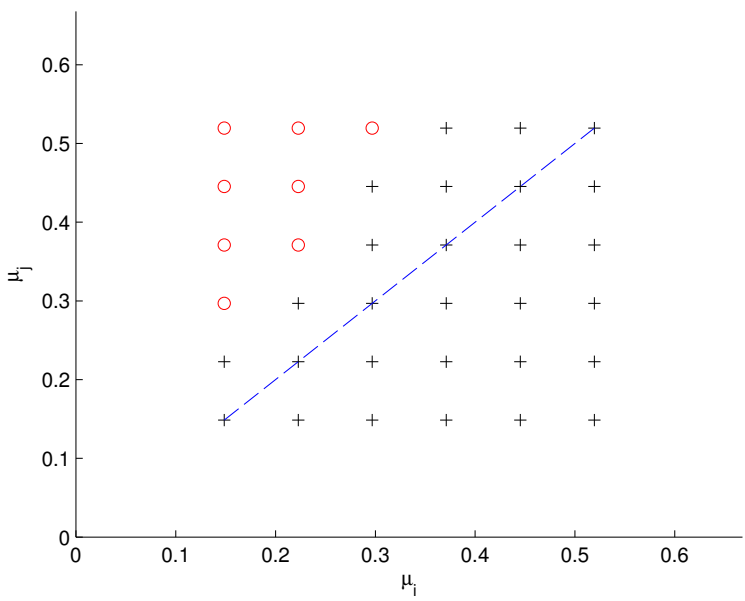

Fig. 6. Ex 1: $\mathcal{R}_{c}$ for $\mu_{i}<\mu_{\max }$ and $i=\{3, \ldots, 8\}$.

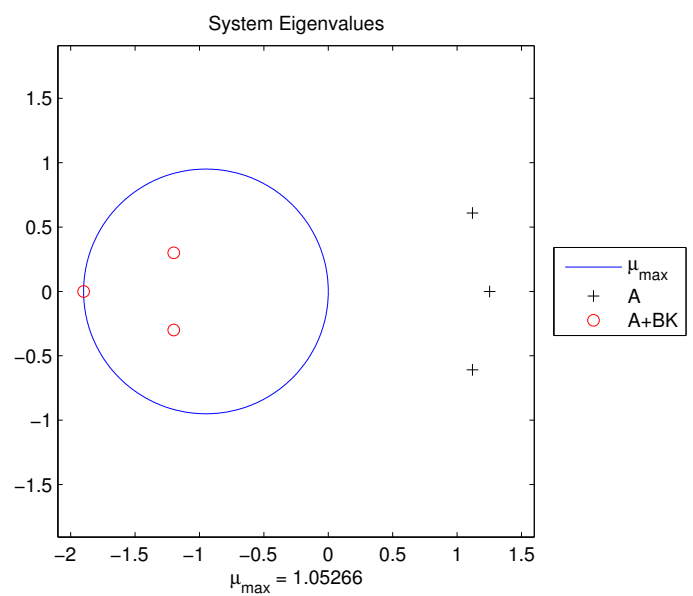

Fig. 7. Ex 2: Eigenvalues of $A$ and $A+B K$ for Example 2. 


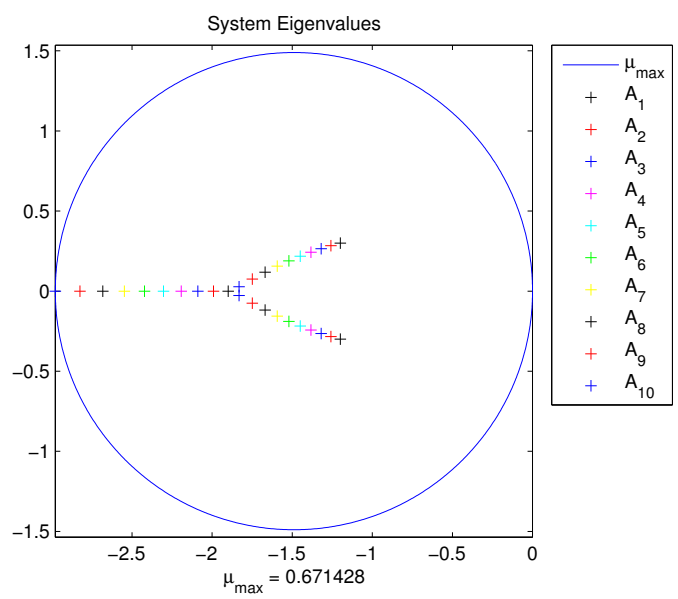

Fig. 8. Ex 2: Eigenvalues of $\mathcal{A}_{i}$ for $\mu_{i}<\mu_{\max }$ and $i=\{1, \ldots, 10\}$.

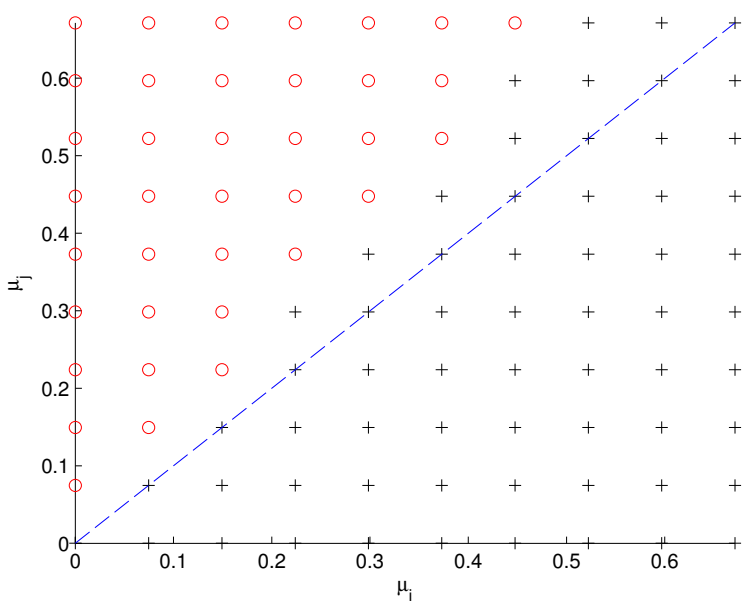

Fig. 9. Ex 2: $\mathcal{R}_{c}$ for $\mu_{i}<\mu_{\max }$ and $i=\{1, \ldots, 10\}$.

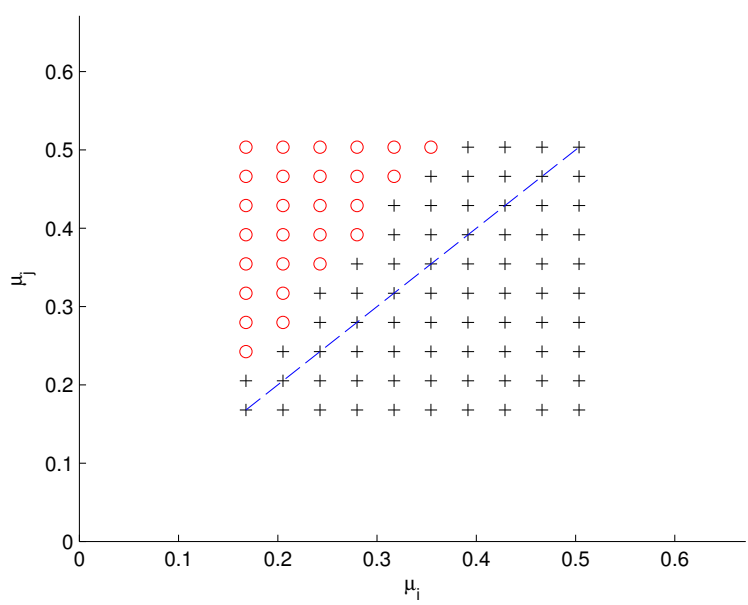

Fig. 10. Ex 2: $\mathcal{R}_{c}$ for $.25 \mu_{\max }<\mu_{i} \leq .75 \mu_{\max }$ and $i=\{1, \ldots, 10\}$.

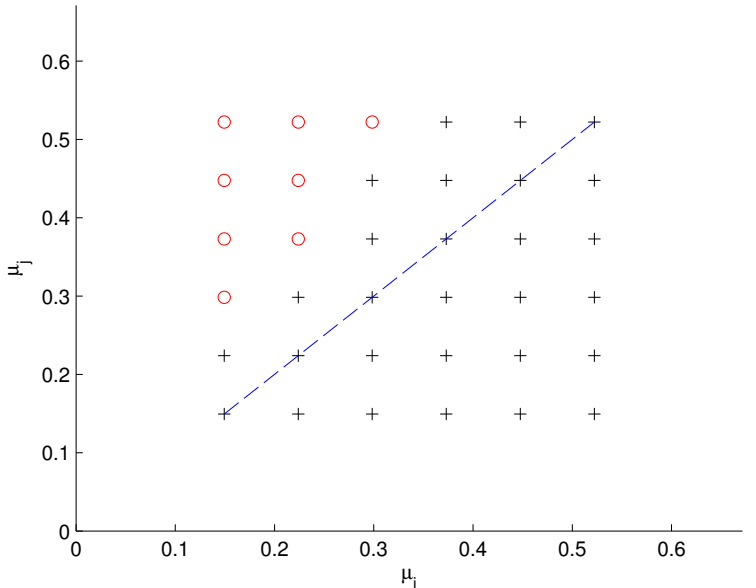

Fig. 11. Ex 2: $\mathcal{R}_{c}$ for $\mu_{i}<\mu_{\max }$ and $i=\{3, \ldots, 8\}$.

discrete time domain, or time scale. The results show that stability of the system depends on both the system and the underlying time scale, and conditions (in the form of a two dimensional region) were given to determine if a particular time scale yeilds stability. When talking about real-time networks, these conditions also give a framework for implementing adaptive sampling by giving the system designer bounds on the sampling periods.

Further research in this area could include application to other specific constrained switching problems, mixed time scales (with continuous and discerete parts), and time-varying linear systems (which might build on [30]), as well as using a multiple Lyapunov function method.

\section{ACKNOWLEDGMENTS}

This work was supported by NSF award CMMI-726996.

\section{REFERENCES}

[1] R. Bishop and R. Dorf, Modern Control Systems, 9th ed. Upper Saddle River, NJ: Prentice-Hall, 2001.

[2] C.-T. Chen, Linear System Theory and Design, 3rd ed. New York, NY: Oxford University Press, 1999

[3] W. Levine, Ed., The Control Handbook. Boca Raton, FL: CRC Press, Inc., 1996.

[4] A. N. Michel, L. Hou, and D. Liu, Stability of Dynamical Systems. Boston, MA: Birkhäuser, 2008.

[5] I. A. Gravagne, J. M. Davis, and J. J. DaCuhna, "A unified approach to high-gain adaptive controllers," Abstract and Appl. Anal., to be published.

[6] I. A. Gravagne, J. M. Davis, J. J. DaCunha, and R. J. Marks II, "Bandwidth reduction for controller area networks using adaptive sampling," in Proc. Int. Conf. Robotics and Automation, New Orleans, LA, Apr. 2004, pp. 5250-5255.

[7] I. A. Gravagne, J. M. Davis, and R. J. Marks II, "How deterministic must a real-time controller be?" in Proc. 2005 IEEE/RSJ Int. Conf. Intell. Robots and Sys., Alberta, Canada, Aug. 2005, pp. 3856-3861.

[8] J. Seiffertt, S. Sanyal, and D. Wunsch, "Hamilton-jacobi-bellman equations and approximate dynamic programming on time scales," IEEE Trans. Sys. Man Cybern. B, Cybern., vol. 38, pp. 918-923, 2008.

[9] R. J. Marks II, I. A. Gravagne, J. M. Davis, and J. J. DaCunha, "Nonregressivity in switched linear circuits and mechanical systems," Math. Comput. Model., vol. 43, pp. 1383-1392, 2006.

[10] Z. Bartosiewicz and E. Pawłuszewicz, "Realizations of linear control systems on time scales," Control \& Cybern., vol. 35, 2006. 
[11] - "Realizations of nonlinear control systems on time scales," IEEE Trans. Autom. Control, vol. 53, 2008.

[12] J. J. DaCunha, "Stability for time varying linear dynamic systems on time scales," J. Comput. Appl. Math., vol. 176, no. 2, pp. 381-410, Apr. 2005 .

[13] J. M. Davis, I. A. Gravagne, B. J. Jackson, and R. J. Marks II, "Controllability, observability, realizability, and stability of dynamic linear systems," Electron. J. Diff. Eq., vol. 2009, pp. 1-32, 2009.

[14] E. Pawłuszewicz and Z. Bartosiewicz, "Linear control systems on time scales: unification of continuous and discrete," in Proc. 10th IEEE Int. Conf. Methods and Models in Automation and Robotics MMAR'04, Miedzyzdroje, Poland, Aug 30 - Sep 22004.

[15] - "Realizability of linear control systems on time scales," in Proc. 11th IEEE Int. Conf. on Methods and Models in Automation and Robotics MMAR'05, Miedzyzdroje, Poland, Aug 29 - Sep 12005.

[16] R. J. Marks II, I. A. Gravagne, and J. M. Davis, "A generalized fourier transform and convolution on time scales," J. Math. Anal. Appl., vol. 340, pp. 901-919, 2008.

[17] J. M. Davis, I. A. Gravagne, B. J. Jackson, R. J. Marks II, and A. A. Ramos, "The laplace transform on time scales revisited," J. Math. Anal. Appl., vol. 332, pp. 1291-1306, 2007.

[18] S. Bhamidi, S. Evans, R. Peled, and P. Ralph, "Brownian motion on disconnected sets, basic hypergeometric functions, and some continued fractions of ramanujan," IMS Collect. Prob. Stat., vol. 2, pp. 42-75, 2008.

[19] K. Zhuang, "Periodic solutions for a stage-structure ecological model on time scales," Electron. J. Diff. Eq., vol. 2007, pp. 1-7, 2007.

[20] F. Atici, D. Biles, and A. Lebedinsky, "An application of time scales to economics," Math. Comput. Model., vol. 43, pp. 718-726, 2006.

[21] F. Atici and F. Uysal, "A production-inventory model of hmms on time scales," Appl. Math. Lett., vol. 21, pp. 236-243, 2008.

[22] D. Liberzon, Switching in Systems and Control. Boston, MA: Birkhäuser, 2003.

[23] A. A. Ramos, "Stability of hybrid dynamical systems: Analysis and design," Ph.D. dissertation, Baylor University, Aug. 2009.

[24] J. E. Miller and I. A. Gravagne, "Stability of simultaneously triangularizable switched systems on time scales," in Proc. 2009 ASEE GulfSouthwest Annu. Conf. American Society for Engineering Education, 2009.

[25] J. E. Miller, "Stability of non-diagonalizable switched linear systems on time scales," Master's thesis, Baylor University, Aug. 2009.

[26] [Online]. Available: www.timescales.org

[27] R. Agarwal, M. Bohner, D. O'Regan, and A. Peterson, "Dynamic equations on time scales: a survey," J. Comput. Appl. Math., vol. 141, pp. 1-26, 2002.

[28] M. Bohner and A. Peterson, Dynamic Equations On Time Scales: An Introduction With Applications. Boston, MA: Birkhäuser, 2001.

[29] M. Bohner and A. Peterson, Eds., Advances in Dynamic Equations On Time Scales. Boston, MA: Birkhäuser, 2003.

[30] J. Zhu and C. Johnson, "Unified canonical forms for matrices over a differential ring," Lin. Algebra and its Appl., vol. 147, pp. 201-248, Mar. 1991. 